\title{
CHANGES IN MATERIAL SYMMETRY ASSOCIATED WITH DEFORMATION: UNIAXIAL EXTENSION
}

\author{
A. S. WINEMAN, ${ }^{1}$ K. R. RAJAGOPAL ${ }^{2}$ and M. NEGAHBAN ${ }^{1}$ \\ ${ }^{1}$ Department of Mechanical Engineering and Applied Mechanics, University of Michigan, Ann \\ Arbor, MI 48109, U.S.A. \\ ${ }^{2}$ Department of Mechanical Engineering, University of Pittsburgh, Pittsburgh, PA 15261, U.S.A.
}

(Communicated by C. G. Speziale)

\begin{abstract}
When a material which is isotropic with respect to a reference configuration is deformed, it is anisotropic with respect to the deformed configuration. The nature of the anisotropy depends on the deformation. In this paper, we consider the special case of the change of a reference configuration by uniaxial extension. We first discuss the structure of the new material symmetry group, and show that it includes non-orthogonal unimodular transformations. Then using the general representation for the response functional of an isotropic material, we show how to construct a representation of the new response functional which satisfies the restrictions of the new material symmetry group.
\end{abstract}

\section{INTRODUCTION}

When a material which is isotropic with respect to a reference placement is deformed, it is anisotropic with respect to the deformed configuration. The nature of the anisotropy depends on the deformation, and Noll's theorem ( $c f$. Truesdell and Noll [1]) provides us with the method for generating the new material symmetry group. Though Noll's theorem has long been available, it has not been gainfully exploited in determining the new material symmetry groups and the corresponding representations for the constitutive functionals. For instance, it is commonly assumed that when a material which is isotropic with respect to a reference placement is uniaxially extended, it would be transversely isotropic with respect to the deformed placement. This common perception is however incorrect. $\dagger$ An application of Noll's theorem shows that the new material symmetry group includes non-orthogonal transformations which are unimodular. In fact, Truesdell [2] anticipated this to be the case in his pictorial representation of the various symmetry groups which arise in the study of material symmetry.

Thus, there is an inherent distinction between a material which is transversely isotropic, and one that was initially isotropic and then uniaxially extended. Due to this distinction in the material symmetry group, the respresentation for the constitutive functions would be different. The correct representation can be obtained by employing the results of Wineman and Pipkin [3].

In this paper, we consider the special case of the change of a reference configuration by uniaxial extension for isotropic materials. We discuss the structure of the new material symmetry group and how the new constitutive representations for the response functionals are deduced.

To understand the relevance of the proposed study, let us consider a material which is isotropic with respect to some reference configuration, and whose constitutive equation is known over the entire domain of possible deformations from this configuration. It is possible, in this case, that the response of the material can be studied without introducing a new reference configuration. The issues discussed in this paper will then play no role in this study.

However, there may be applications in which it is convenient to change to a new reference configuration. For example, in metal plasticity, a stress-free deformed state is often considered as a possible new reference configuration. Among other quantities associated with this state, one would like to know the yield function, which will depend on the new symmetry group associated with this state. The results of this study will then prove useful.

† More precisely, in addition to (2.14) being satisfied for all $\mathbf{G}$ which belong to the material symmetry group corresponding to transverse isotropy, it also is satisfied for other $\mathbf{G}$ which do not belong to the transverse isotropy group, some of which are non-orthogonal. 
We discuss next, another possible application where the present study may be of use. Suppose a material is isotropic with respect to some reference configuration, and its constitutive equation is known over only part of the domain of possible deformations from this configuration. One may attempt to extend the domain of definition of the constitutive equation by first introducing a new reference configuration. A "continuation" of the constitutive equation could possibly be constructed for deformations from this new configuration. If this "continuation" is developed in a manner consistent with the ideas discussed in this paper, it will be a proper continuation of the original continuation of the original constitutive equation. Of course, the precise nature of this "continuation" depends on the choice of the class of materials under consideration. While these ideas are yet vague and in a nascent state, it is important to recognize that the study might provide the basic building blocks for such an analysis.

The underlying concepts associated with a change of reference configuration, and relations between response functionals and material symmetry groups, are reviewed in Section 2. The most general representation of a constitutive equation for isotropic simple solids is introduced in Section 3. Its transformation associated with a change of reference configuration is then derived. In Section 4, Noll's theorem is used to study the structure of the new material symmetry group associated with the new configuration. A method is introduced, in Section 5, for generating the constitutive response functional appropriate to the new configuration from that corresponding to the original one. Using the notion of anisotropic tensors $(c f .[4,5])$, it is shown that the new constitutive response functional can be written in a manner which is formally similar to one for transverse isotropy, and yet meets the restrictions associated with the non-orthogonal unimodular transformations of the new symmetry group. These ideas are illustrated in Section 6 for a specific class of constitutive functions, namely those for nonlinear elasticity.

\section{NOTATION, BASIC RELATIONS}

Let $\boldsymbol{\kappa}(\mathscr{B})$ denote a reference configuration for an isotropic simple solid, and let $\boldsymbol{K}^{*}(\mathscr{B})$ denote a second configuration. In this paper, $\kappa^{*}(\mathscr{B})$ is regarded as any possible configuration of the body. It need not coincide with a configuration actually taken by the body. For example, $\kappa^{*}(\mathscr{B})$ could represent a state of permanent deformation taken by the solid if the stresses were removed. It could also be a base state on which additional deformation is superposed.

The positions of a given material particle in reference configurations $\kappa(\mathscr{B})$ and $\kappa^{*}(\mathscr{B})$ are denoted by $\mathbf{X}$ and $\mathbf{X}^{*}$, respectively. The two reference configurations are related by

$$
\mathbf{X}^{*}=\Omega(\mathbf{X}), \text { where } \Omega=\kappa^{*} \circ \kappa^{-1} \text {. }
$$

If $\mathbf{x}(\tau)$ denotes the position of a particle at time $\tau$, its motion is described by expressions of the form

$$
\begin{aligned}
& \mathbf{x}(\tau)=\chi(\mathbf{X}, \tau), \quad \mathbf{X} \in K(\mathscr{B}), \\
& \mathbf{x}(\tau)=\chi^{*}\left(\mathbf{X}^{*}, \tau\right), \quad \mathbf{X}^{*} \in K^{*}(\mathscr{B}) .
\end{aligned}
$$

The deformation gradients associated with the descriptions (2.2) and (2.3) are denoted, respectively, by $\mathbf{F}(\tau)$ and $\mathbf{F}^{*}(\tau)$. Their components with respect to a Cartesian coordinate system are given by

These are related by

$$
F(\tau)_{i j}=\partial x_{i}(\tau) / \partial X_{j}, \quad F^{*}(\tau)_{i j}=\partial x_{i}(\tau) / \partial X_{j}^{*}
$$

$$
\mathbf{F}(\tau)=\mathbf{F}^{*}(\tau) \mathbf{P},
$$

where $\mathbf{P} \equiv \partial \Omega / \partial \mathbf{X}$ is a transformation whose components are given by

$$
P_{i i}=\partial X_{i}^{*} / \partial X_{j}
$$

The stress $\sigma(t)$ at a material particle at time $t$ is given in terms of the deformation gradient 
history $\mathbf{F}(\tau),-\infty<\tau \leqslant t$, by a constitutive equation of the form

$$
\boldsymbol{\sigma}(t)=F_{K}[\mathbf{F} \underset{\tau=-\infty}{(t)}]
$$

In relation (2.7), $\mathscr{F}_{k}$ denotes a response functional whose definition is associated with the use of reference placement $\kappa(\mathscr{B})$. In a similar manner, if the motion is referred to reference configuration $K^{*}(\mathscr{B})$, the constitutive equation involves a response functional $\mathscr{F}_{\kappa^{*}}$, and

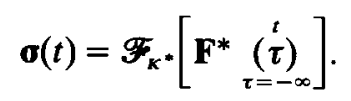

For notational convenience, the range of $\tau$ will no longer be indicated on the response functionals. The response functional $\mathscr{F}_{K^{*}}$ can be expressed in terms of the response functional $\mathscr{F}_{k}$ by using relation $(2.5)(c f .[1])$,

$$
\mathscr{F}_{K^{*}}\left[\mathbf{F}^{*}(\tau)\right]=\mathscr{F}_{k}\left[\mathbf{F}^{*}(\tau) \mathbf{P}\right]
$$

In this work, we shall not make any extraneous assumptions, either about the class of functions which are admissible as histories, or about the nature of the response functionals $\mathscr{F}_{k}$, or $F_{\kappa^{*}}$. The response functional is to be regarded as any rule of correspondence which specifies $\boldsymbol{\sigma}(t)$ when the history $\mathbf{F}(\tau)$, or $\mathbf{F}^{*}(\tau)$ is given.

It is a consequence of the principle of material frame indifference [1] that the response functional $\mathscr{F}_{k}$ can be written in the form

where

$$
\mathscr{F}_{\kappa}[\mathbf{F}(\tau)]=\mathbf{F}(t) \mathscr{R}_{\kappa}[\mathbf{C}(\tau)] \mathbf{F}(t)^{\mathrm{T}}
$$

$$
\mathbf{C}(\tau)=\mathbf{F}(\tau)^{\mathrm{T}} \mathbf{F}(\tau)
$$

and $\mathscr{R}_{\kappa}$ denotes another functional associated with reference configuration $\kappa(\mathscr{B})$. In a similar manner,

where

$$
\mathscr{F}_{\kappa} \cdot\left[\mathbf{F}^{*}(\tau)\right]=\mathbf{F}^{*}(t) \mathscr{R}_{\kappa^{*}}\left[\mathbf{C}^{*}(\tau)\right] \mathbf{F}^{*}(t)^{\mathrm{T}}
$$

$$
\mathbf{C}^{*}(\tau)=\mathbf{F}^{*}(\tau)^{\mathrm{T}} \mathbf{F}^{*}(\tau)
$$

Let $g_{\kappa}$ denote the group of material symmetry transformations associated with reference configuration $\kappa(\mathscr{B})$. Then for each transformation $\mathbf{G} \in g_{\kappa}$,

$$
\mathscr{F}_{\kappa}[\mathbf{F}(\tau)]=\mathscr{F}_{\kappa}[\mathbf{F}(\tau) \mathbf{G}]
$$

Similarly, let $g_{\kappa^{*}}$ denote the group of material symmetry transformations associated with $\boldsymbol{K}^{*}(\mathscr{B})$. For each transformation $\mathbf{G}^{*} \in g_{\boldsymbol{K}^{*}}$,

$$
\mathscr{F}_{\mathrm{K}^{*}}\left[\mathbf{F}^{*}(\tau)\right]=\mathscr{F}_{\mathrm{K}^{*}}\left[\mathbf{F}^{*}(\tau) \mathbf{G}^{*}\right]
$$

It has been shown by Noll [1] that

$$
g_{\kappa^{*}}=\mathbf{P} g_{\kappa} \mathbf{P}^{-1}
$$

\section{ISOTROPIC SIMPLE SOLIDS}

Suppose that the material is isotropic in reference configuration $\kappa(\mathscr{B})$, so that the material symmetry group $g_{\kappa}$ is the orthogonal group (full or proper). Then eqn (2.14) must be satisfied identically in the history $\mathbf{F}(\tau)$ for each transformation $\mathbf{G}$ satisfying the orthogonality condition

$$
\mathbf{G}^{\mathrm{T}} \mathbf{G}=\mathbf{G G}^{\mathrm{T}}=\mathbf{I} \text {. }
$$

According to the representation theorem of Wineman and Pipkin [2] applied to isotropic materials, the response functional $\mathscr{R}_{k}$, related to $\mathscr{F}_{k}$ by eqn $(2.10)$, can be written in the form

$$
\mathscr{R}_{\kappa}=\sum_{\alpha=0}^{5} \mathscr{L}_{\kappa}^{(\alpha)}\left[\mathbf{J}^{(\alpha)} ; I\right],
$$


where $\mathscr{L}_{x}^{(\alpha)}$ denotes a functional which depends on a matrix polynomial $\mathbf{J}^{(\alpha)}$, and on a set of invariants $I$. The functional $\mathscr{L}_{\kappa}^{(\alpha)}$ satisfies the following property in its matrix polymomial argument $\mathrm{J}^{(\alpha)}$ : if $\phi_{i j}$ is a constant matrix, then

$$
\mathscr{L}_{\kappa}^{(\alpha)}\left(\phi_{i j} J_{i j} ; I\right)=\phi_{i j} \mathscr{L}_{\kappa}^{(\alpha)}\left[J_{i j} ; I\right] .
$$

The set of invariants denoted by $I$ consists of the following:

$$
\begin{aligned}
& I^{(1)}(\zeta)=\operatorname{tr} \mathbf{C}(\zeta), \\
& I^{(2)}\left(\zeta_{1}, \zeta_{2}\right)=\operatorname{tr} \mathbf{C}\left(\zeta_{1}\right) \mathbf{C}\left(\zeta_{2}\right), \\
& I^{(\beta)}\left(\zeta_{1}, \zeta_{2}, \ldots, \zeta_{\beta}\right)=\operatorname{tr} \mathbf{C}\left(\zeta_{1}\right) \mathbf{C}\left(\zeta_{2}\right) \cdots \mathbf{C}\left(\zeta_{\beta}\right) ; \quad \beta=1,2, \ldots, 6
\end{aligned}
$$

The matrix polynomials $\mathbf{J}^{(\alpha)}$ are defined by

$$
\begin{aligned}
\mathbf{J}^{(0)} & =\mathbf{I}, \\
\mathbf{J}^{(\alpha)} & =\frac{1}{2}\left(\boldsymbol{\pi}^{(\alpha)}+\boldsymbol{\pi}^{(\alpha) \mathrm{T}}\right), \quad \alpha=1,2, \ldots, 5, \\
\boldsymbol{\pi}^{(\alpha)} & =\mathbf{C}\left(\zeta_{1}\right) \mathbf{C}\left(\zeta_{2}\right) \cdots \mathbf{C}\left(\zeta_{\alpha}\right) .
\end{aligned}
$$

Recall that the response functional $\mathscr{F}_{K^{*}}$ associated with reference configuration $\kappa^{*}(\mathscr{B})$ can be expressed in terms of $\mathscr{F}_{k}$ by means of eqn (2.9). That is, $\mathscr{F}_{K^{*}}$ can be constructed from $\mathscr{F}_{k}$ by making the substitution defined by eqn (2.5). To this end, we substitute for eqn (2.5) into eqn (2.11) and then make use of eqn (2.13) to obtain

$$
\mathbf{C}(\tau)=\mathbf{P}^{\mathrm{T}} \mathbf{C}^{*}(\tau) \mathbf{P}
$$

When eqn (3.6) is used in eqns (3.4), the invariants of set $I$ become of the form

$$
I^{(\beta)}\left(\zeta_{1}, \zeta_{2}, \ldots, \zeta_{\beta}\right)=\operatorname{tr} \mathbf{C}^{*}\left(\zeta_{1}\right) \mathscr{B}_{0} \mathbf{C}^{*}\left(\zeta_{2}\right) \mathscr{B}_{0} \cdots \mathbf{C}^{*}\left(\zeta_{\beta}\right) \mathscr{B}_{0} ; \quad \beta=1,2, \ldots, 6,
$$

where

$$
\mathscr{B}_{0}=\mathbf{P P}^{\mathrm{T}} \text {. }
$$

Denote the set of invariants in this form as $\tilde{I}$. When eqn (3.6) is substituted into eqns (3.5), the matrix polynomials $\mathbf{J}^{(\alpha)}(\alpha=1,2, \ldots, 5)$ become

where

$$
\mathbf{J}^{(\alpha)}=\mathbf{P}^{\mathrm{T}} \tilde{\mathbf{J}}^{(\alpha)} \mathbf{P},
$$

$$
\tilde{\mathbf{J}}^{(\alpha)}=\frac{1}{2}\left(\tilde{\pi}^{(\alpha)}+\tilde{\pi}^{(\alpha) \mathrm{T}}\right)
$$

with

$$
\tilde{\boldsymbol{\pi}}^{(\alpha)}=\mathbf{C}^{*}\left(\zeta_{1}\right) \mathscr{B}_{0} \mathbf{C}^{*}\left(\zeta_{2}\right) \mathscr{B}_{0} \cdots \mathscr{B}_{0} \mathbf{C}^{*}\left(\zeta_{\alpha}\right)
$$

Let eqns (2.5), (3.2), (3.7) and (3.8) be combined in eqn (2.10) to give

$$
\mathscr{F}_{\kappa}\left[\mathbf{F}^{*}(\tau) \mathbf{P}\right]=\mathbf{F}^{*}(t) \mathbf{P}\left\{\mathscr{L}_{\kappa}^{(0)}[\mathbf{I} ; \tilde{I}]+\sum_{\alpha=1}^{5} \mathscr{L}_{\kappa}^{(\alpha)}\left[\mathbf{P}^{\mathrm{T}} \tilde{\mathbf{J}}^{(\alpha)} \mathbf{P} ; \tilde{I}\right]\right\} \mathbf{P}^{\mathrm{T}} \mathbf{F}^{*}(t)^{\mathrm{T}}
$$

Since $\mathbf{P}$, defined by eqn (2.5), is a constant tensor, the properties of $\mathscr{L}_{\boldsymbol{k}}^{(\alpha)}$ defined in eqn (3.3) imply that eqn (3.12) can be written as

$$
\mathscr{F}_{\kappa}\left[\mathbf{F}^{*}(\tau) \mathbf{P}\right]=\mathbf{F}^{*}(t)\left\{\mathscr{L}_{\kappa}^{(0)}\left[\mathbf{P} \mathbf{P}^{\mathrm{T}} ; \tilde{I}\right]+\sum_{\alpha=1}^{5} \mathscr{L}_{\kappa}^{(\alpha)}\left[\mathbf{P P}^{\mathrm{T}} \tilde{J}^{(\alpha)} \mathbf{P} \mathbf{P}^{\mathrm{T}} ; \tilde{I}\right]\right\} \mathbf{F}^{*}(t)^{\mathrm{T}}
$$

Finally, by eqns (2.9) and (3.8), we obtain

$$
\mathscr{F}_{\kappa^{*}}\left[\mathbf{F}^{*}(\tau)\right]=\mathbf{F}^{*}(t)\left\{\mathscr{L}_{\kappa}^{(0)}\left[\mathscr{B}_{0} ; \tilde{I}\right]+\sum_{\alpha=1}^{5} \mathscr{L}_{\kappa}^{(\alpha)}\left[\mathscr{B}_{0} \tilde{\mathbf{J}}^{(\alpha)} \mathscr{B}_{0} ; \tilde{I}\right]\right\} \mathbf{F}^{*}(t)^{\mathrm{T}}
$$

It will be important in the remainder of this section to recall how the invariants in the set $\tilde{I}$ and the matrix polynomials $\tilde{\mathbf{J}}^{(\alpha)}$ depend on $\mathbf{C}^{*}(\zeta)$ and $\mathscr{B}_{0}$. In order to emphasize the definitions in eqns (3.7) and (3.11), we introduce the notation

$$
\tilde{I}=\tilde{I}\left(\mathbf{C}^{*}, \mathscr{B}_{0}\right), \tilde{\mathbf{J}}^{(\alpha)}=\tilde{\mathbf{J}}^{(\alpha)}\left(\mathbf{C}^{*}, \mathscr{B}_{0}\right), \tilde{\boldsymbol{\pi}}^{(\alpha)}=\tilde{\pi}^{(\alpha)}\left(\mathbf{C}^{*}, \mathscr{B}_{0}\right) .
$$


We now consider the material symmetry transformations associated with configuration $\boldsymbol{K}^{*}(\mathscr{B})$. If $\mathbf{G}^{*}$ is to be a transformation, it must satisfy eqn (2.15) identically in the history $\mathbf{F}^{*}(\tau)$. In order to study the properties of $\mathbf{G}^{*}$, it will be useful to calculate the right-hand side of eqn (2.15) by substituting $\mathbf{F}^{*}(\tau) \mathbf{G}^{*}$ for $\mathbf{F}^{*}(\tau)$ in eqn (3.14). First note, by eqn (2.13), $\mathbf{C}^{*}$ becomes $\mathbf{G}^{* \mathrm{~T}} \mathbf{C}^{*} \mathbf{G}^{*}$. The invariants in eqn (3.7) can be written as

$$
I^{(\beta)}\left(\zeta_{1}, \zeta_{2}, \ldots, \zeta_{\beta}\right)=\operatorname{tr} \mathbf{C}^{*}\left(\zeta_{1}\right) \hat{\hat{B}_{0}} \mathbf{C}^{*}\left(\zeta_{2}\right) \hat{\hat{B}_{0}} \cdots \mathbf{C}^{*}\left(\zeta_{\beta}\right) \hat{\hat{B}_{0}}
$$

where

$$
\hat{\mathscr{B}}_{0}=\mathbf{G}^{*} \mathscr{B}_{0} \mathbf{G}^{* T} \text {. }
$$

A comparison of eqns (3.7) and (3.16) shows that $\mathscr{B}_{0}$ is replaced by $\hat{\mathscr{B}}_{0}$. In terms of the notation introduced in eqn (3.15), the result of the substitution can be written as

$$
\tilde{I}\left(\mathbf{C}^{*}, \mathscr{B}_{0}\right) \rightarrow \tilde{I}\left(\mathbf{C}^{*}, \hat{\mathscr{B}}_{0}\right) \text {. }
$$

In a similar manner, we can write for the matrix polynomial $\tilde{\pi}^{(\alpha)}$ defined in (3.11),

$$
\tilde{\boldsymbol{\pi}}^{(\alpha)}\left(\mathbf{C}^{*}, \boldsymbol{B}_{0}\right) \rightarrow \mathbf{G}^{* \mathrm{~T}} \mathbf{C}^{*}\left(\zeta_{1}\right) \hat{\mathscr{B}}_{0} \mathbf{C}^{*}\left(\zeta_{2}\right) \hat{\mathscr{B}}_{0} \ldots \hat{\mathscr{B}}_{0} \mathbf{C}^{*}\left(\zeta_{\alpha}\right) \mathbf{G}^{*}=\mathbf{G}^{* \mathrm{~T}} \tilde{\boldsymbol{\pi}}^{(\alpha)}\left(\mathbf{C}^{*}, \hat{\mathfrak{B}}_{0}\right) \mathbf{G}^{*}
$$

By eqns (3.10) and (3.11), the matrix polynomial argument in eqn (3.14) becomes

$$
\mathscr{B}_{0} \tilde{\mathbf{J}}^{(\alpha)}\left(\mathbf{C}^{*}, \mathscr{B}_{0}\right) \mathscr{B}_{0} \rightarrow \mathscr{B}_{0} \mathbf{G}^{* \mathrm{~T}} \tilde{\mathbf{J}}^{(\alpha)}\left(\mathbf{C}^{*}, \hat{\mathscr{B}_{0}}\right) \mathbf{G}^{*} \mathscr{B}_{0}
$$

It then follows that

$$
\begin{aligned}
\mathscr{F}_{\boldsymbol{k}} \cdot\left[\mathbf{F}^{*}(\tau) \mathbf{G}^{*}\right]= & \mathbf{F}^{*}(t)\left\{\mathbf{G}^{*} \mathscr{L}_{\kappa}^{(0)}\left[\mathscr{B}_{0} ; \tilde{I}\left(\mathbf{C}^{*}, \hat{\mathscr{B}_{0}}\right)\right] \mathbf{G}^{* \mathrm{~T}}\right. \\
& \left.+\sum_{\alpha=1}^{5} \mathbf{G}^{*} \mathscr{L}_{\boldsymbol{k}}^{(\alpha)}\left[\mathscr{B}_{0} \mathbf{G}^{* \mathrm{~T}} \tilde{J}^{(\alpha)}\left(\mathbf{C}^{*}, \hat{\mathscr{B}}_{0}\right) \mathbf{G}^{*} \mathscr{B}_{0} ; I\left(\mathbf{C}^{*}, \hat{\hat{B}_{0}}\right)\right] \mathbf{G}^{* \mathrm{~T}}\right\} \mathbf{F}^{*}(t)
\end{aligned}
$$

Since $\mathbf{G}$ is a constant tensor, property (3.3) of $\mathscr{L}_{\kappa}^{(\alpha)}$ and eqn (3.17) imply

$$
\begin{aligned}
\mathscr{F}_{\boldsymbol{K}} \cdot\left[\mathbf{F}^{*}(\tau) \mathbf{G}^{*}\right]= & \mathbf{F}^{*}(t)\left\{\mathscr{L}_{\kappa}^{(0)}\left[\hat{\mathscr{B}}_{0} ; \tilde{I}\left(\mathbf{C}^{*}, \hat{\mathscr{B}}_{0}\right)\right]\right. \\
& \left.+\sum_{\alpha=1}^{5} \mathscr{L}_{\kappa}^{(\alpha)}\left[\hat{\mathscr{B}}_{0} \tilde{J}^{(\alpha)}\left(\mathbf{C}^{*}, \hat{\mathscr{B}_{0}}\right) \hat{\mathscr{B}_{0}} ; \tilde{I}\left(\mathbf{C}^{*}, \hat{\mathscr{B}}_{0}\right)\right]\right\} \mathbf{F}^{*}(t)^{\mathrm{T}} .
\end{aligned}
$$

A comparison of eqns (3.14) and (3.22) shows that $\mathscr{F}_{K^{*}}\left[\mathbf{F}^{*}(\tau) \mathbf{G}^{*}\right]$ is obtained from $\mathscr{F}_{k^{*}}\left[\mathbf{F}^{*}(\tau)\right]$ by replacing $\mathscr{B}_{0}$ by $\hat{\mathscr{B}}_{0}$. If $\mathbf{G}^{*}$ is to be a material symmetry transformation associated with $\boldsymbol{K}^{*}(\mathscr{B})$, then eqn (2.15) must be satisfied. This will occur if $\mathscr{B}_{0}=\mathscr{B}_{0}$ in eqn (3.17).

\section{UNIAXIAL OR EQUAL BIAXIAL STRETCH}

We now consider the case in which configuration $\kappa^{*}(\mathscr{B})$ is related to configuration $\kappa(\mathscr{B})$ by a deformation of the following form with respect to a Cartesian coordinate system,

$$
\begin{aligned}
& X_{1}^{*}=\lambda X_{1}, \\
& X_{2}^{*}=\lambda X_{2}, \\
& X_{3}^{*}=\mu X_{3} .
\end{aligned}
$$

This corresponds to a unixial stretch along the $X_{3}$-axis or an equal biaxial stretch in the $X_{1}-X_{2}$ plane. By eqn (2.6)

$$
\mathbf{P}=\left[\begin{array}{lll}
\lambda & 0 & 0 \\
0 & \lambda & 0 \\
0 & 0 & \mu
\end{array}\right]
$$

The material symmetry group associated with $\kappa^{*}(\mathscr{B})$ which is induced by the above deformation from the material symmetry group of configuration $\kappa(\mathscr{B})$ can be calculated using Noll's Theorem (2.16). Let $\mathbf{G}=\left[G_{i j}\right]$ be an orthogonal transformation in the group $g_{\kappa}$. The corresponding symmetry transformation $\mathbf{G}^{*}$ of $g_{\kappa^{*}}$, by eqns $(2.16)$ and (4.2), has the matrix 
representation

where

$$
\mathbf{G}^{*}=\left[\begin{array}{rrl}
G_{11} & G_{12} & G_{13} / \omega \\
G_{21} & G_{22} & G_{23} / \omega \\
\omega G_{31} & \omega G_{32} & G_{33}
\end{array}\right]
$$

$$
\omega=\mu / \lambda
$$

Consider a rotation about, say, the $X_{3}$-axis, which can be represented by the matrix

$$
\mathbf{G}=\left[\begin{array}{cll}
\cos \theta & \sin \theta & 0 \\
-\sin \theta & \cos \theta & 0 \\
0 & 0 & 1
\end{array}\right], \quad 0 \leqslant \theta \leqslant 2 \pi .
$$

Also consider the reflection transformations

$$
\left[\begin{array}{rrr}
-1 & 0 & 0 \\
0 & 1 & 0 \\
0 & 0 & 1
\end{array}\right], \quad\left[\begin{array}{rrr}
1 & 0 & 0 \\
0 & 1 & 0 \\
0 & 0 & -1
\end{array}\right], \quad\left[\begin{array}{rrr}
-1 & 0 & 0 \\
0 & 1 & 0 \\
0 & 0 & -1
\end{array}\right] \text {. }
$$

There are five possible cases of transverse isotropy which can be generated by transformations of the form (4.5) together with one or more of the transformations (4.6) (cf. Spencer [4]). For any $\mathbf{G}$ of the form (4.5), or which is the product of the rotation (4.5) with one of the transformations (4.6), it is seen from (4.3) that $\mathbf{G}^{*}=\mathbf{G}$.

Now, suppose that $\mathbf{G}$ is an orthogonal transformation which is not of the form produced by products of (4.5) and (4.6). Some of its components $G_{13}, G_{23}, G_{31}, G_{32}$ are non-zero. As seen from eqn (4.3), some of the corresponding components of $\mathbf{G}^{*}$ are not zero, and are multiplied by $\omega$. Since, according to (4.1) and (4.4), $\omega \neq 1$, it follows that $\mathbf{G}^{*}$ will not satisfy (3.1) and therefore will be a unimodular, non-orthogonal transformation. As an example, suppose that $\mathbf{G}$ is a rotation about the $X_{1}$-axis. Then

$$
\mathbf{G}=\left[\begin{array}{ccc}
1 & 0 & 0 \\
0 & \cos \phi & \sin \phi \\
0 & -\sin \phi & \cos \phi
\end{array}\right]
$$

By eqn (2.16), the corresponding transformation $\mathbf{G}^{*}$ in $g_{\kappa^{*}}$ has the form

$$
\mathbf{G}^{*}\left[\begin{array}{cccc}
1 & 0 & 0 & \\
0 & \cos \phi & \omega^{-1} & \sin \phi \\
0 & -\omega \sin \phi & \cos \phi &
\end{array}\right]
$$

which is clearly non-orthogonal, but unimodular.

Thus, the symmetry group $g_{\kappa^{*}}$ contains the transformations of transverse isotropy as a subgroup, and, in addition, contains unimodular non-orthogonal transformations. It follows that a material which is transversely isotropic with reference to a placement is inherently different from a material which was originally isotropic with regard to a reference placement and subsequently uniaxially extended. This difference must be considered when developing representations for constitutive equations in the two cases.

\section{MATERIAL SYMMETRY RESTRICTION ON THE RESPONSE FUNCTIONAL}

Equations (2.14) and (2.15) impose restrictions on the response functionals $\mathscr{F}_{k}$ and $\mathscr{F}_{k^{*}}$, respectively, due to material symmetry. These functionals must be such that eqns (2.14) and (2.15) are satisfied identically in the deformation gradient for each transformation of the material symmetry group. The general method for constructing representations for response functionals which meet the restrictions of eqns (2.14) and (2.15) has been outlined by Wineman and Pipkin [3]. 
Since the material symmetry group $g_{x^{*}}$ contains the transverse isotropy subgroup, one might be tempted, using the method outlined in [3], to write a respresentation for the response functional $\mathscr{F}_{x^{*}}$ for a material which is transversely isotropic. However, the appropriate representation for $\mathscr{F}_{x^{*}}$. must also satisfy eqn (2.15) for the unimodular non-orthogonal transformations in $g_{\kappa^{*}}$. This shows that the representation of a response functional with regard to the deformed configuration of a material which has been uniaxially extended would be different from that employed for a transversely isotropic material.

From another point of view, the representations for response functionals $\mathscr{F}_{K}$ and $\mathscr{F}_{k}$ cannot be independent. Equation (2.9) shows that $\mathscr{F}_{K^{*}}$ is related to $\mathscr{F}_{K}$, and $\mathscr{F}_{K^{*}}$ must reduce to $\mathscr{F}_{K}$ if $\lambda=\mu=1$ in eqn (4.1). The presence of unimodular, non-orthogonal transformations in $g_{\kappa^{*}}$, in the example treated here, arises from the connection of $g_{\kappa^{*}}$ to $g_{\kappa}$ by eqn (2.16). By incorporating the restrictions associated with the unimodular, non-orthogonal transformations in the representation, the response functional $\mathscr{F}_{K^{*}}$ will have the proper connection to $\mathscr{F}_{\mathrm{K}}$.

In this section, we establish a method for constructing a representation for $\mathscr{F}_{\kappa}$ * which has the same structure as one for transverse isotropy, but which also satisfies the restrictions imposed by the unimodular, non-orthogonal symmetry transformations.

By eqn (4.2) and (3.7)

$$
\mathscr{B}_{0}=\left[\begin{array}{lll}
\lambda^{2} & 0 & 0 \\
0 & \lambda^{2} & 0 \\
0 & 0 & \mu^{2}
\end{array}\right] .
$$

Let $\boldsymbol{\alpha}$ and $\boldsymbol{\beta}$ be tensors whose components are defined by

$$
\begin{aligned}
& \alpha_{i j}=\delta_{3 i} \delta_{3 j}, \\
& \beta_{i j}=\delta_{1 i} \delta_{1 j}+\delta_{2 i} \delta_{2 j}=\delta_{\alpha i} \delta_{\alpha j},
\end{aligned}
$$

where the repeated Greek indices indicate summation over the range 1 and 2 . Then, $\mathscr{B}_{0}$ can be written as

or in the equivalent form

$$
\begin{aligned}
\mathscr{B}_{0} & =\lambda^{2} \mathbf{I}+\left(\mu^{2}-\lambda^{2}\right) \boldsymbol{\alpha} \\
& =\lambda^{2}\left[\mathbf{I}+\left(\omega^{2}-1\right) \boldsymbol{\alpha}\right],
\end{aligned}
$$

$$
\begin{aligned}
\mathscr{B}_{0} & =\lambda^{2} \boldsymbol{\beta}+\mu^{2} \boldsymbol{\alpha} \\
& =\lambda^{2}\left[\boldsymbol{\beta}+\omega^{2} \boldsymbol{\alpha}\right],
\end{aligned}
$$

where $\omega$ was defined in eqn (4.5).

Smith and Rivlin [5] have shown that I, $\alpha$ and $\boldsymbol{\beta}$ are anisotropic tensors for the transverse isotropy group of transformations. That is,

$$
\begin{aligned}
\mathbf{G}^{*} \mathbf{I} \mathbf{G}^{* \mathbf{T}} & =\mathbf{I}, \\
\mathbf{G}^{*} \boldsymbol{\alpha} \mathbf{G}^{* \mathbf{T}} & =\boldsymbol{\alpha}, \\
\mathbf{G}^{*} \boldsymbol{\beta} \mathbf{G}^{* \mathbf{T}} & =\boldsymbol{\beta},
\end{aligned}
$$

if $\mathbf{G}^{*}$ is any of the transformations (4.5) or (4.6). It follows that when $\mathscr{B}_{0}$ is given by eqns (5.4) or (5.5),

$$
\mathbf{G}^{*} \mathscr{B}_{0} \mathbf{G}^{* \mathrm{~T}}=\mathscr{B}_{0}
$$

for any transformation of the transverse isotropy group. Then by eqns $(3.17) \mathscr{\mathscr { B }}_{0}=\mathscr{B}_{0}$, and according to eqns (3.14) and (3.22)

$$
\mathscr{F}_{\mathrm{K}} \cdot\left[\mathbf{F}^{*}(\tau)\right]=\mathscr{F}_{\mathrm{K}^{*}}\left[\mathbf{F}^{*}(\tau) \mathbf{G}^{*}\right]
$$

This demonstrates, in the context of the present example, the result of Noll's thoerem that the transverse isotropy group is a subgroup of $g_{\mathrm{x}}$ *-

Now let $\mathbf{G}^{*}$ be the unimodular, non-orthogonal transformation given by eqn (4.8). It is a straightforward calculation to show that relations (5.6) are no longer satisfied, but again $\mathscr{S}_{0}=\mathscr{B}_{0}$. Noll's theorem assures that this will be the case for all of the unimodular transformations in $g_{\kappa}^{*}$. Thus $\mathscr{B}_{0}=\mathscr{B}_{0}$ for all $\mathbf{G}^{*}$ in $\mathrm{g}_{\kappa}^{*}$. 
The expansions (5.4) or (5.5) of $\mathscr{B}_{0}$ in terms of the anisotropic tensors $\mathbf{I}, \boldsymbol{\alpha}$ and $\boldsymbol{\beta}$ have an important application. Each of these expansions can be used to construct a representation for $\mathscr{F}_{K^{*}}$ which has the formal structure appropriate to the transverse isotropy subgroup of $g_{K^{*}}$, but which also includes the restrictions due to the unimodular non-orthogonal transformations of $g_{\kappa^{*}}$.

\section{Representation I}

Let $\mathscr{B}_{0}$ given by eqn (5.4) be substituted into the invariants defined in (3.7). The result is

$$
I^{(\beta)}\left(\zeta_{1}, \zeta_{2}, \ldots, \zeta_{\beta}\right)=\lambda^{2 \beta}\left[I^{(\beta)}\left(\zeta_{1}, \zeta_{2}, \ldots, \zeta_{\beta}\right)+\Delta P^{(\beta)}\left(\zeta_{1}, \zeta_{2}, \ldots, \zeta_{\beta} ; \Delta\right)\right.
$$

where

$$
\stackrel{*}{I}(\beta)^{(\beta)}\left(\zeta_{1}, \zeta_{2}, \ldots, \zeta_{\beta}\right)=\operatorname{tr} \mathbf{C}^{*}\left(\zeta_{1}\right) \mathbf{C}^{*}\left(\zeta_{2}\right) \cdots \mathbf{C}^{*}\left(\zeta_{\beta}\right) ; \quad \beta=1,2, \ldots, 6,
$$

$\Delta=\omega^{2}-1$ and $P^{(\beta)}$ is a polynomial in $\Delta$ and the expressions

$$
\begin{aligned}
K^{*(\beta)}\left(\zeta_{1}, \zeta_{2}, \ldots, \zeta_{\beta}\right) & =\left[\mathbf{C}^{*}\left(\zeta_{1}\right) \mathbf{C}^{*}\left(\zeta_{2}\right) \cdots \mathbf{C}^{*}\left(\zeta_{\beta}\right)\right]_{33} \\
& =C^{*}\left(\zeta_{1}\right)_{3 i_{1}} C^{*}\left(\zeta_{2}\right)_{i_{1} i_{2}} \cdots C^{*}\left(\zeta_{\beta}\right)_{i_{\beta-1} 3} ; \quad \beta=1,2, \ldots, 6 .
\end{aligned}
$$

For notational convenience, denote the set of expressions in (5.10) and (5.11) by $K^{*}$.

Upon substitution of $\mathscr{B}_{0}$, the matrix polynomial $\mathscr{B}_{0} \tilde{\mathbf{J}}^{(\alpha)} \mathscr{B}_{0},(\alpha=1,2, \ldots, 5)$, can be written in the form

$$
\mathscr{B}_{0} \tilde{\mathbf{J}}^{(\alpha)} \mathscr{B}_{0}=\lambda^{2(\alpha+1)}\left[\mathbf{J}^{*}(\alpha)+\Delta \sum_{\gamma=6}^{N_{\alpha}} f_{\alpha \gamma}\left(K^{*}, \Delta\right) \mathbf{J}^{*}(\gamma)\right]
$$

where

$$
\begin{gathered}
\mathbf{J}^{(\alpha)}=\frac{1}{2}\left(*^{(\alpha)}+*^{(\alpha) \mathrm{T}}\right), \\
\boldsymbol{\pi}^{(\alpha)}=\mathbf{C}^{*}\left(\zeta_{1}\right) \mathbf{C}^{*}\left(\zeta_{2}\right) \cdots \mathbf{C}^{*}\left(\zeta_{\alpha}\right) ; \quad \alpha=1,2, \ldots, 5,
\end{gathered}
$$

$f_{\alpha \gamma}$ is a scalar polynomial in $\Delta$ and the elements of the set $K^{*}$, and $\mathbf{J}^{(\alpha)}, \alpha=6, \ldots, N_{\alpha}$, are matrix polynomials which are the symmetric parts of

$$
\begin{gathered}
\delta_{3 i} \delta_{3 j} \\
\delta_{3 i}\left[\pi^{(\nu)}\right]_{3 j} ; \quad \gamma=1,2, \ldots, \alpha-1, \\
{\left[\pi^{*}\left(\gamma_{1}\right)\right]_{3 i}\left[\pi^{\left(\gamma_{2}\right)}\right]_{3 j}, \quad \gamma_{1}, \gamma_{2}=1,2, \ldots, \alpha ; \quad \gamma_{1}+\gamma_{2} \leqslant \alpha .}
\end{gathered}
$$

For example, for $\beta=3$ in eqn (3.7),

$$
\begin{aligned}
I^{(3)}\left(\zeta_{1}, \zeta_{2}, \zeta_{3}\right)= & \lambda^{6}\left\{I^{*}(3)\left(\zeta_{1}, \zeta_{2}, \zeta_{3}\right)+\Delta\left[C^{*}\left(\zeta_{1}\right)_{3 j} C^{*}\left(\zeta_{2}\right)_{j k} C^{*}\left(\zeta_{3}\right)_{k 3}\right.\right. \\
& \left.+C^{*}\left(\zeta_{3}\right)_{3 j} C^{*}\left(\zeta_{1}\right)_{j k} C^{*}\left(\zeta_{2}\right)_{k 3}+C^{*}\left(\zeta_{2}\right)_{3 j} C^{*}\left(\zeta_{3}\right)_{j k} C^{*}\left(\zeta_{1}\right)_{k 3}\right] \\
& +\Delta^{2}\left[C^{*}\left(\zeta_{1}\right)_{3 j} C^{*}\left(\zeta_{2}\right)_{j 3} C^{*}\left(\zeta_{3}\right)_{33}+C^{*}\left(\zeta_{2}\right)_{3 j} C^{*}\left(\zeta_{3}\right)_{j 3} C^{*}\left(\zeta_{1}\right)_{33}\right. \\
& \left.\left.+C^{*}\left(\zeta_{3}\right)_{3 j} C^{*}\left(\zeta_{1}\right)_{j 3} C^{*}\left(\zeta_{2}\right)_{33}\right]+\Delta^{3} C^{*}\left(\zeta_{1}\right)_{33} C^{*}\left(\zeta_{2}\right)_{33} C^{*}\left(\zeta_{3}\right)_{33}\right\}
\end{aligned}
$$

where $\Delta=\omega^{2}-1$. Also, for $\alpha=2$ in eqn (3.11),

$$
\begin{aligned}
{\left[\mathscr{B}_{0} \mathbf{C}\left(\zeta_{1}\right) \mathscr{B}_{0} \mathbf{C}\left(\zeta_{2}\right) \mathscr{B}_{0}\right]_{i j}=} & \lambda^{6}\left\{C^{*}\left(\zeta_{1}\right)_{i p} C\left(\zeta_{2}\right)_{p j}\right. \\
& +\Delta\left[C^{*}\left(\zeta_{1}\right)_{i 3} C\left(\zeta_{2}\right)_{j 3}+\delta_{3 i} C^{*}\left(\zeta_{1}\right)_{3 k} C^{*}\left(\zeta_{2}\right)_{k j}+\delta_{3 j} C^{*}\left(\zeta_{1}\right)_{i p} C^{*}\left(\zeta_{2}\right)_{p 3}\right] \\
& +\Delta^{2}\left[\delta_{3 i} C^{*}\left(\zeta_{2}\right)_{3 j} C^{*}\left(\zeta_{1}\right)_{33}+\delta_{3 j} C^{*}\left(\zeta_{1}\right)_{3 i} C^{*}\left(\zeta_{2}\right)_{33}\right. \\
& \left.\left.+\delta_{3 i} \delta_{3 j} C^{*}\left(\zeta_{1}\right)_{3 p} C^{*}\left(\zeta_{2}\right)_{p 3}\right]+\Delta^{3} \delta_{3 i} \delta_{3 j} C^{*}\left(\zeta_{1}\right)_{33} C^{*}\left(\zeta_{2}\right)_{33}\right\}
\end{aligned}
$$

The scalar polynomials in eqns (5.10) and (5.11) were derived by Adkins [6] as invariants associated with transverse isotropy for second order tensors. By comparison with eqn (3.4), it is seen that eqns (5.10) are also invariants for isotropy. Thus, the invariants in eqn (5.11) are 
additional ones arising from transverse isotropy. This set is reducible, but that reduction will not be considered here. The matrix polynomials in (5.13) are the same as in eqn (3.5) for isotropy. The ones in (5.14) are additional ones which arise from transverse isotropy.

When eqns (5.4) and (5.12) are substituted into eqn (3.14), and use is made of its property (3.3), we obtain

$$
\begin{aligned}
\mathscr{F}_{\kappa^{*}}\left[\mathbf{F}^{*}(\tau)\right]= & \mathbf{F}^{*}(t)\left\{\mathscr{L}_{\kappa^{*}}^{(0)}\left[\mathbf{I} ; K^{*}, \Delta\right]+\sum_{\alpha=1}^{5} \mathscr{L}_{\kappa^{*}}^{(\alpha)}\left[\mathbf{J}^{(\alpha)} ; K^{*}, \Delta\right]\right. \\
& \left.+\sum_{\alpha=6}^{N^{*}} \mathscr{L}_{\kappa^{*}}^{(\alpha)}\left[\mathbf{J}^{*}(\alpha) ; K^{*}, \Delta\right]\right\} \mathbf{F}^{*}(t)^{\mathrm{T}}
\end{aligned}
$$

where $\mathscr{L}_{\kappa^{*}}^{(\alpha)}$ are new functionals which are expressible in terms of the original functionals $\mathscr{L}_{\kappa}^{(\alpha)}$.

Note that when $\mu=\lambda$, i.e. equal triaxial extension $\Delta=\omega^{2}-1=0$, and $\mathscr{B}_{0}=\lambda^{2} \mathbf{I}$. Then the terms which are associated with transverse isotropy disappear from eqns (5.9) and (5.11). Thus, $\Delta=(\mu / \lambda)^{2}-1$ can be interpreted as a measure of the amount of transverse isotropy which is induced by deformation (4.1). The functionals $\mathscr{L}_{\kappa^{*}}^{(\alpha)}$ also satisfy property (3.3). Equation (5.17) gives the same form that would be obtained for transversely isotropic materials by the direct application of the method outlined in [3]. In that method, the functionals $\mathscr{L}_{\mathrm{K}^{* *}}^{(\alpha)}$ depend on invariants $K^{*}$ in an arbitrary manner. The response functional would satisfy eqn (2.15) only for the transformations of transverse isotropy. When one uses the method presented here, one is assured that the new functionals $\mathscr{L}_{\kappa^{*}}^{(\alpha)}$ are such that eqn (2.15) is satisfied for all the transformations of $g_{\kappa^{*}}$.

\section{Representation $I I$}

Let $\mathscr{B}_{0}$ given by eqn (5.5) be substituted into the invariants (3.7). These can be expressed as polynomials in $\omega^{2}$ and the polynomials of the set

$$
\begin{aligned}
& C_{\alpha \alpha}^{*}(\tau), \quad C_{33}^{*}(\tau), \\
& C_{\alpha \beta}^{*}\left(\tau_{1}\right) C_{\alpha \beta}^{*}\left(\tau_{2}\right), \quad C_{3 \alpha}^{*}\left(\tau_{1}\right) C_{\alpha 3}^{*}\left(\tau_{2}\right), \\
& C_{3 \alpha}^{*}\left(\tau_{1}\right) C_{\alpha \beta}^{*}\left(\tau_{2}\right) C_{\beta 3}^{*}\left(\tau_{3}\right), \\
& C_{3 \alpha}^{*}\left(\tau_{1}\right) C_{\alpha \beta}^{*}\left(\tau_{2}\right) C_{\beta \gamma}^{*}\left(\tau_{3}\right) C_{\gamma 3}^{*}\left(\tau_{4}\right),
\end{aligned}
$$

in which the arguments $\tau_{1}, \tau_{2}, \tau_{3}, \tau_{4}$ are replaced by various combinations of $\zeta_{1}, \zeta_{2}, \ldots, \zeta_{6}$. According to Adkins [6] and Spencer [4], set (5.18) is an irreducible set of invariants for second-order tensors which is appropriate to transverse isotropy.

Each matrix polynomial $\mathscr{B}_{0} J^{(\alpha)} \mathscr{B}_{0}$ can be written in the form

$$
\mathscr{B}_{o} \mathbf{J}^{(\alpha)} \mathscr{B}_{0}=\sum_{\gamma=1}^{M_{\alpha}} f^{(\alpha)} \mathbf{J}^{\prime^{\prime(\alpha)}}
$$

where $f^{\prime(\alpha)}$ is a scalar polynomial in the elements of the set (5.18), and $\mathbf{J}^{\prime(\alpha)}, \alpha=1, M_{\alpha}$ are matrix polynomials which are the symmetric parts of

$$
\begin{aligned}
& \alpha_{i j}, \quad \beta_{i j} \\
& \delta_{i \alpha} \delta_{j \beta} C_{\alpha \beta}^{*}(\tau) \\
& \delta_{i 3} \delta_{j \alpha} C_{\alpha 3}^{*}(\tau) \\
& \delta_{i \alpha} C_{\alpha 3}^{*}\left(\tau_{1}\right) \delta_{j \beta} C_{\beta 3}^{*}\left(\tau_{2}\right) \\
& \delta_{i 3} \delta_{j \alpha} C_{\alpha \beta}^{*}\left(\tau_{1}\right) C_{\beta 3}^{*}\left(\tau_{2}\right) \\
& \delta_{i 3} \delta_{j \alpha} C_{\alpha \beta}^{*}\left(\tau_{1}\right) C_{\beta \gamma}^{*}\left(\tau_{2}\right) C_{\gamma 3}^{*}\left(\tau_{3}\right) \\
& \delta_{i \alpha} C_{\alpha 3}^{*}\left(\tau_{1}\right) \delta_{j \beta} C_{\beta \gamma}^{*}\left(\tau_{2}\right) C_{\gamma 3}^{*}\left(\tau_{3}\right) .
\end{aligned}
$$

The algebra required to express the invariants and matrix polynomials in eqn (3.14) in terms of $(5.10)$ and $(5.20)$ is quite tedious. For this reason, no examples are presented here. When 
eqn (5.5) and (5.19) are substituted into eqn (3.14), we obtain

$$
\mathscr{F}_{K^{*}}\left[\mathbf{F}^{*}(\tau)\right]=\mathbf{F}^{*}(t) \sum_{\alpha=1}^{M^{*}} \mathscr{L}_{\mathbf{K}^{*}}^{\prime(\alpha)}\left[\mathbf{J}^{\prime \prime(\alpha)} ; K^{* \prime}, \omega^{2}\right] \mathbf{F}^{*}(t)^{\mathrm{T}},
$$

where $K^{* \prime}$ denotes the invariants generated by eqn (5.18). The discussion following eqn (5.17) also applies here. This is an alternate representation which is completely equivalent to that in eqn (5.17).

\section{EXAMPLE: NONLINEAR ELASTICITY}

It will be instructive to illustrate the previous discussion for nonlinear elasticity. In this case, the response functional $\mathscr{R}_{\kappa}$ in eqn (2.10) has the form (cf. [1])

$$
\mathscr{R}_{\kappa}[\mathbf{F}(\tau)]=\frac{\rho}{\rho_{0}}\left(\frac{\partial W}{\partial \mathbf{C}}+\frac{\partial W}{\partial \mathbf{C}^{\mathrm{T}}}\right)
$$

where $W$ denotes the strain energy density function and $W=W(\mathbf{C}(t))$. For notational convenience, we suppress the explicit dependence on current time $t$ and write $\mathbf{C}=\mathbf{C}(t)$.

If the material is isotropic in reference configuration $\kappa(\mathscr{B})$, then $W=\bar{W}\left(I_{1}, I_{2}, I_{3}\right)$, where

$$
\begin{aligned}
& I_{1}=\operatorname{tr} \mathbf{C}, \\
& I_{2}=\frac{1}{2}\left(I_{1}^{2}-\operatorname{tr} \mathbf{C}^{2}\right), \\
& I_{3}=\operatorname{det} \mathbf{C} .
\end{aligned}
$$

The response functional $\mathscr{R}_{\mathrm{K}}$ in (6.1) becomes

where

$$
\mathscr{R}_{\kappa}[\mathbf{F}(\tau)]=\bar{\Phi}_{0} \mathbf{I}+\bar{\Phi}_{1} \mathbf{C}+\bar{\Phi}_{2} \mathbf{C}^{2}
$$

and

$$
\bar{\Phi}_{\alpha}=\bar{\Phi}_{\alpha}\left(I_{1}, I_{2}, I_{3}\right), \quad \alpha=1,2,3,
$$

$$
\begin{aligned}
& \bar{\Phi}_{0}=2 \frac{\rho}{\rho_{0}}\left(\frac{\partial \bar{W}}{\partial I_{1}}+I_{1} \frac{\partial \bar{W}}{\partial I_{2}}+I_{2} \frac{\partial \bar{W}}{\partial I_{3}}\right), \\
& \bar{\Phi}_{1}=-2 \frac{\rho}{\rho_{0}}\left(\frac{\partial \bar{W}}{\partial I_{2}}+I_{1} \frac{\partial \bar{W}}{\partial I_{3}}\right), \\
& \bar{\Phi}_{2}=2 \frac{\rho}{\rho_{0}} \frac{\partial \bar{W}}{\partial I_{3}} .
\end{aligned}
$$

The expression in eqn (6.3) is a special case of the general representation for isotropic materials in eqn (3.2) in which

$$
\begin{gathered}
\mathscr{L}_{\kappa}^{(0)}=\overline{\boldsymbol{\Phi}}_{0} \mathbf{I}, \quad \mathscr{L}_{\kappa}^{(1)}=\overline{\boldsymbol{\Phi}}_{1} \mathbf{C}, \quad \mathscr{L}_{\kappa}^{(2)}=\overline{\boldsymbol{\Phi}}_{2} \mathbf{C}^{2} \\
\mathscr{L}_{\kappa}^{(\beta)}=0, \quad \beta=3,4,5 .
\end{gathered}
$$

If the material is transversely isotropic in reference configuration $\kappa(\mathscr{B})$, then $W=$ $\tilde{W}\left(I_{1}, I_{2}, I_{3}, K_{1}, K_{2}\right)$, where $I_{1}, I_{2}, I_{3}$ are defined in eqn (6.2), and

$$
\begin{aligned}
& K_{1}=C_{33}, \\
& K_{2}=\left(\mathbf{C}^{2}\right)_{33}=C_{3 p} C_{3 p} .
\end{aligned}
$$

When this form of $W$ is substituted into eqn (6.1), and use is made of eqns (6.2) and (6.6), the response functional $\mathscr{R}_{\kappa}$ has the form

$$
\mathscr{R}_{\kappa}=\tilde{\phi}_{0} \mathbf{I}+\tilde{\phi}_{1} \mathbf{C}+\tilde{\phi}_{2} \mathbf{C}^{2}+\tilde{\phi}_{3} \boldsymbol{\alpha}+\tilde{\phi}_{4} \mathbf{D}
$$


where $\boldsymbol{\alpha}$ is defined in eqn (5.2), and $\mathbf{D}$ has components defined by

$$
D_{i j}=C_{3 i} \delta_{3 j}+C_{3 j} \delta_{3 i} \text {. }
$$

In addition, $\tilde{\phi}_{\beta}=\tilde{\phi}_{\beta}\left(I_{1}, I_{2}, I_{3}, K_{1}, K_{2}\right), \beta=0,1, \ldots, 4$, and

$$
\begin{aligned}
& \tilde{\phi}_{0}=2 \frac{\rho}{\rho_{0}}\left(\frac{\partial \tilde{W}}{\partial I_{1}}+I_{1} \frac{\partial \tilde{W}}{\partial I_{2}}+I_{2} \frac{\partial \tilde{W}}{\partial I_{3}}\right), \\
& \tilde{\phi}_{1}=-2 \frac{\rho}{\rho_{0}}\left(\frac{\partial \tilde{W}}{\partial I_{2}}+I_{1} \frac{\partial \tilde{W}}{\partial I_{3}}\right), \\
& \tilde{\phi}_{2}=2 \frac{\rho}{\rho_{0}} \frac{\partial \tilde{W}}{\partial I_{3}}, \quad \tilde{\phi}_{3}=2 \frac{\rho}{\rho_{0}} \frac{\partial \tilde{W}}{\partial K_{1}}, \quad \tilde{\phi}_{4}=2 \frac{\rho}{\rho_{0}} \frac{\partial \tilde{W}}{\partial K_{2}}
\end{aligned}
$$

Now, let $F_{K}$ be transformed into $\mathscr{F}_{K^{*}}$ by the substitution $\mathbf{F}=\mathbf{F}^{*} \mathbf{P}$, as defined in eqn (2.9). Then $\mathscr{F}_{K}$ in eqn $(2.10)$ becomes $\mathscr{F}_{K^{*}}$ in eqn (2.12), where by eqn (3.6) and (3.8), the corresponding form of $\mathscr{R}_{\mathrm{K}}$ * is given by

$$
\mathscr{R}_{\boldsymbol{\kappa}^{*}}=\tilde{\phi}_{0} \mathscr{B}_{0}+\tilde{\phi}_{1} \mathscr{B}_{0} \mathbf{C}^{*} \mathscr{B}_{0}+\tilde{\phi}_{2} \mathscr{B}_{0} \mathbf{C}^{*} \mathscr{B}_{0} \boldsymbol{C}^{*} \mathscr{B}_{0}
$$

When $\mathscr{R}_{\mathrm{x}^{*}}$ in eqn (6.10) is substituted into eqn (2.12), the result is the special case of (3.14) for isotropic nonlinear elastic materials. The arguments of $\tilde{\phi}_{\beta}, \beta=0,1,2$ become, by eqn (3.2),

$$
\begin{aligned}
& I_{1}=\operatorname{tr} \mathbf{C}^{*} \mathscr{B}_{0}, \\
& I_{2}=\frac{1}{2}\left[I_{1}^{2}-\operatorname{tr} \mathbf{C}^{*} \mathscr{B}_{0} C^{*} \mathscr{B}_{0}\right], \\
& I_{3}=\operatorname{det} \mathbf{C}^{*} \cdot \operatorname{det} \mathscr{B}_{0} .
\end{aligned}
$$

Let $K^{*}(\mathscr{B})$ be related to $K(\mathscr{B})$ by a uniaxial extension, as discussed in Section 5 . We will now show that $\mathscr{R}_{\mathrm{K}^{*}}$ can be rewritten so that it has the same mathematical form as $\mathscr{R}_{\mathrm{k}}$ for a transversely isotropic material, given by eqn (6.7). This will correspond to Representation $I$ of Section 5 . To this end, let $\mathscr{B}_{0}$ be given by eqn (5.4). Upon substitution of this form of $\mathscr{B}_{0}$ into the expressions for the invariants in eqns (6.10), they can be rewritten in the form

$$
\begin{aligned}
& I_{1}=\lambda^{2}\left[I_{1}^{*}+\left(\omega^{2}-1\right) K_{1}^{*}\right], \\
& I_{2}=\lambda^{4}\left[I_{2}^{*}+\left(\omega^{2}-1\right)\left(I_{1}^{*} K_{1}^{*}-K_{2}^{*}\right)\right], \\
& I_{3}=\lambda^{6} \omega^{2} I_{3}^{*} .
\end{aligned}
$$

In eqn (6.12), $I_{1}^{*}, I_{2}^{*}, I_{3}^{*}, K_{1}^{*}, K_{2}^{*}$ are invariants which are defined in eqns (6.2) and (6.6) with $\mathrm{C}$ replaced by $\mathbf{C}^{*}$.

The matrices in eqn (6.10) are given by eqn (5.4) and

$$
\begin{gathered}
\mathscr{B}_{0} \mathbf{C}^{*} \mathscr{B}_{0}=\lambda^{4}\left[\mathbf{C}^{*}+\left(\omega^{2}-1\right) \mathbf{D}^{*}+\left(\omega^{2}-1\right)^{2} K_{1}^{*} \boldsymbol{\alpha}\right], \\
\mathscr{B}_{0} \mathbf{C}^{*} \mathscr{B}_{0} \mathbf{C}^{*} \mathscr{B}_{0}=\lambda^{6}\left[\mathbf{C}^{* 2}+\left(\omega^{2}-1\right) \mathbf{E}^{*}+\left(\omega^{2}-1\right)^{2}\left(K_{1}^{*} \mathbf{D}^{*}+K_{2}^{*} \boldsymbol{\alpha}\right)+\left(\omega^{2}-1\right)^{3}\left(K_{1}^{*}\right)^{2} \boldsymbol{\alpha}\right] .
\end{gathered}
$$

In the above, the components of $\mathbf{D}^{*}$ are defined as in eqn (6.8), with $\mathbf{C}$ replaced by $\mathbf{C}^{*}$. The components of $\mathbf{E}^{*}$ are defined by

$$
E_{i j}^{*}=\left(C^{*}\right)_{3 i}^{2} \delta_{3 j}+\left(C^{*}\right)_{3 j}^{2} \delta_{3 i}+C_{3 i}^{*} C_{3 j}^{*}
$$

The matrix $\mathbf{E}^{*}$ can be expressed in terms of the other matrices in the following manner. Note that

$$
\frac{\partial\left(C^{*}\right)_{33}^{3}}{\partial C_{i j}}=E_{i j}^{*}
$$

Recall that by the Cayley-Hamilton theorem,

$$
\left(C^{*}\right)_{33}^{3}=\left(C^{*}\right)_{23}^{2} I_{1}^{*}-C_{33}^{*} I_{2}+I_{3}^{*} \text {. }
$$


When eqn (6.17) is substituted into eqn (6.16), the result is

$$
\mathbf{E}^{*}=\left(K_{2}^{*}-K_{1}^{*} I_{1}^{*}+I_{2}^{*}\right) \mathbf{I}+\left(K_{1}^{*}-I_{1}^{*}\right) \mathbf{C}^{*}+\left(\mathbf{C}^{*}\right)^{2}-I_{2}^{*} \boldsymbol{\alpha}+I_{1}^{*} \mathbf{D}^{*} .
$$

Let eqn (5.4), (6.12), (6.13), (6.14) and (6.18) be combined in eqn (6.10). The resulting expression for $\mathscr{R}_{\mathrm{k}}$ * can be written as

$$
\mathscr{R}_{\boldsymbol{K}^{*}}=\Psi_{0}^{*} \mathbf{I}+\psi_{1}^{*} \mathbf{C}^{*}+\Psi_{2}^{*} \mathbf{C}^{* 2}+\Psi_{3}^{*} \boldsymbol{\alpha}+\Psi_{4}^{*} \mathbf{D}^{*},
$$

where the scalar coefficients are defined by the expressions

$$
\begin{aligned}
\Psi_{0}^{*}= & \phi_{0}^{*} \lambda^{2}+\phi_{2}^{*} \lambda^{6}\left(\omega^{2}-1\right)\left(K_{2}^{*}-K_{1}^{*} I_{1}^{*}+I_{2}^{*}\right), \\
\Psi_{1}^{*}= & \phi_{1}^{*} \lambda^{4}+\phi_{2}^{*} \lambda^{6}\left(\omega^{2}-1\right)\left(K_{1}^{*}-I_{1}^{*}\right), \\
\Psi_{2}^{*}= & \phi_{2}^{*} \lambda^{6} \omega^{2}, \\
\Psi_{3}^{*}= & \phi_{0}^{*} \lambda^{2}\left(\omega^{2}-1\right)+\phi_{1}^{*} \lambda^{4}\left(\omega^{2}-1\right)^{2} K_{1}^{*} \\
& +\phi_{2}^{*}\left[\left(\omega^{2}-1\right)^{2}\left(K_{2}^{*}\right)^{2}+\left(\omega^{2}-1\right)^{3}\left(K_{1}^{*}\right)^{2}-\left(\omega^{2}-1\right) I_{2}^{*}\right], \\
\Psi_{4}^{*}= & \phi_{1}^{*} \lambda^{4}\left(\omega^{2}-1\right)+\phi_{2}^{*} \lambda^{6}\left[\left(\omega^{2}-1\right)^{2} K_{1}^{*}+\left(\omega^{2}-1\right) I_{1}^{*}\right] .
\end{aligned}
$$

The scalars $\phi_{\beta}^{*}$ are defined by the use of eqn (6.12)

$$
\bar{\phi}_{\beta}\left(I_{1}, I_{2}, I_{3}\right)=\phi_{\beta}^{*}\left(I_{1}^{*}, I_{2}^{*}, I_{3}^{*}, K_{1}^{*}, K_{2}^{*} ; \lambda^{2}, \omega^{2}\right), \quad \beta=0,1, \ldots, 4 .
$$

In view of eqns $(6.20)$ and $(6.21)$, it can be seen that

$$
\psi_{\beta}^{*}=\Psi_{\beta}^{*}\left(I_{1}^{*}, I_{2}^{*}, I_{3}^{*}, K_{1}^{*}, K_{2}^{*} ; \lambda^{2}, \omega^{2}\right) .
$$

A comparison of eqns (6.19) and (6.22) with eqn (6.7) shows that $\mathscr{R}_{\kappa^{*}}$ has the same form as $\mathscr{R}_{\kappa}$ for a transversely isotropic material.

Equation (5.8) will be satisfied for transformations $\mathbf{G}^{*}$ of transverse isotropy. However, the manner of deriving the representation for $\mathscr{R}_{\kappa^{*}}$ ensures that eqn (5.8) will also hold for the unimodular non-orthogonal transformations of the symmetry group $g_{\kappa}=$.

It is essential to note that if $\mathscr{R}_{\kappa^{*}}$ is given by eqn (6.19), with arbitrary scalar coefficients, $\Psi_{\alpha}^{*}=\Psi_{\alpha}^{*}\left(I_{1}^{*}, I_{2}^{*}, I_{3}^{*}, K_{1}^{*}, K_{2}^{*}\right)$, then eqn (5.18) will be satisfied only for transformations $\mathbf{G}^{*}$ associated with transverse isotropy. However, if $\mathscr{R}_{\boldsymbol{k}^{*}}$ is to be regarded as applicable to the deformed configuration of a material which has been uniaxially extended, the scalar coefficients must have the form in eqn (6.20). This emphasizes the fact that material which is transversely isotropic with respect to a placement is inherently different from a material which was originally isotropic with regard to a placement, and was then uniaxially extended.

\section{REFERENCES}

[1] C. TRUESDELL and W. NOLL, The Non-Linear Field Theories of Mechanics. Springer, Berlin (1965).

[2] C. TRUESDELL, Six Lectures on Modern Natural Philosophy. Springer, Berlin (1966).

3] A. S. WINEMAN and A. C. PIPKIN, Archs ratl Mech. Analysis 17, 184 (1964).

[4] A. J. M. SPENCER, in Continuum Physics (Edited by A. C. ERINGEN), Vol. 3. Academic Press, New York (1975).

[5] G. F. SMITH and R. S. RIVLIN, Q. appl. Math. 15, 308 (1957).

[6] J. E. ADKINS, Archs ratl Mech. Analysis 4, 193 (1960). 